\title{
A PROBLEM ON PRODUCTS OF TOEPLITZ OPERATORS
}

\author{
KUN YU GUO
}

(Communicated by Albert Baernstein II)

\begin{abstract}
A natural and interesting problem on classical Hardy space of one complex variable is the following:

Problem: If $T_{\varphi_{1}} T_{\varphi_{2}} \cdots T_{\varphi_{n}}=0$, then there exist some $i$ such that $\varphi_{i}=0$.

In this note, we establish the kernel inclusion theorem for the products of Toeplitz operators. Using this fact, in case $n=5$, we give the above question an affirmative answer.
\end{abstract}

Let $D$ be the open unit disk in the complex plane and $T$ its boundary. For the definitions and related conceptions of Hardy space $H^{2}(T, d \theta / 2 \pi)$ and Toeplitz operators on $H^{2}(T, d \theta / 2 \pi)$, refer to [1, Chapters 6 and 7]; for convenience, write $H^{2}(T, d \theta / 2 \pi)$ as $H^{2}$. To prove the main results in this note, we establish the following lemmas.

Lemma 1. For $g \in L^{\infty}$ and $g \neq 0$, let $E$ be a measurable subset of $T$ with $0<$ meas $E<2 \pi,\left.g\right|_{E}=0$. Then $\operatorname{ker} T_{g}=\{0\}$.

The proof of Lemma 1 is trivial.

Lemma 2. For $f, g \in L^{\infty}$ and $f, g \neq 0$, let $E$ be a measurable subset of $T$ with $0<$ meas $E<2 \pi$ and $\left.g\right|_{E}=0$. If $\operatorname{ker} T_{f} T_{g} \neq\{0\}$, then there exists a function $\psi(\neq 0)$ such that

$$
T_{g} h=\psi h \quad \forall h \in \operatorname{ker} T_{f} T_{g} .
$$

Proof. For $h_{0} \in \operatorname{ker} T_{f} T_{g}, h_{0} \neq 0$, by Lemma 1, there exist $h_{1} \in H^{2}$ and $h_{1} \neq 0$ such that $f T_{g} h_{0}=\overline{h_{1}}$. So, $T_{g} h_{0}=\frac{\overline{h_{1}}}{f}$. It follows that there is $h_{2} \in H^{2}$ such that $g h_{0}=\frac{\overline{h_{1}}}{f}-\overline{h_{2}}$. Hence $\left.\frac{1}{f}\right|_{E}=\left.\left(\frac{\overline{h_{2}}}{h_{1}}\right)\right|_{E}$. Similarly for $h_{0}^{\prime} \in \operatorname{ker} T_{f} T_{g}$ and $h_{0}^{\prime} \neq h_{0}$, there exist $h_{1}^{\prime}, h_{2}^{\prime} \in H^{2}$ such that $\left.\frac{1}{f}\right|_{E}=\left.\left(\frac{\overline{h_{2}^{\prime}}}{h_{1}^{\prime}}\right)\right|_{E}$. Therefore

$$
\left.\left(\overline{\frac{h_{2}}{h_{1}}}\right)\right|_{E}=\left.\left(\overline{\frac{h_{2}^{\prime}}{h_{1}^{\prime}}}\right)\right|_{E}
$$

i.e., $\left.\left(h_{2} h_{1}^{\prime}-h_{2}^{\prime} h_{1}\right)\right|_{E}=0$. Since $h_{2} h_{1}^{\prime}-h_{2}^{\prime} h_{1} \in H^{1}$ and $0<$ meas $E<2 \pi$, we get that $h_{2} h_{1}^{\prime}=h_{2}^{\prime} h_{1}$. Put $h^{\prime}=\frac{h_{2}}{h_{1}}=\frac{h_{2}^{\prime}}{h_{1}^{\prime}}$; according to the preceding reasoning, we

Received by the editors September 20, 1994

1991 Mathematics Subject Classification. Primary 47B35. 
obtain

$$
\begin{aligned}
g h_{0} & =\frac{\overline{h_{1}}}{f}-\overline{h_{2}}=\frac{\overline{h_{1}}}{f}-\overline{h_{1} h^{\prime}}=\overline{h_{1}}\left(\frac{1}{f}-\overline{h^{\prime}}\right) \\
& =f\left(\frac{1}{f}-\overline{h^{\prime}}\right) T_{g} h_{0}=\left(1-f \overline{h^{\prime}}\right) T_{g} h_{0} .
\end{aligned}
$$

Similarly, we have

$$
g h_{0}^{\prime}=\left(1-f \overline{h^{\prime}}\right) T_{g} h_{0}^{\prime} .
$$

Since $\left.\frac{g h_{0}}{g h_{0}^{\prime}}\right|_{T \backslash E}=\left.\frac{h_{0}}{h_{0}^{\prime}}\right|_{T \backslash E}$, the above argument leads to the following:

$$
\frac{h_{0}}{h_{0}^{\prime}}=\frac{T_{g} h_{0}}{T_{g} h_{0}^{\prime}}
$$

i.e., $\frac{T_{g} h_{0}}{h_{0}}=\frac{T_{g} h_{0}^{\prime}}{h_{0}^{\prime}}$. Set $\psi=\frac{T_{g} h_{0}}{h_{0}}$; clearly such a $\psi$ is independent on the choice of $h_{0}$ in the $\operatorname{ker} T_{f} T_{g}$. Thus the following is true:

$$
T_{g} h=\psi h \quad \forall h \in \operatorname{ker} T_{f} T_{g} .
$$

This completes the proof of Lemma 1 .

Next, we establish the kernel inclusion theorem, which is interesting in itself.

Theorem 1. Under the same assumption as in Lemma 2, there exist $\varphi \in L^{\infty}$ and $\varphi \neq 0$ such that

$$
\operatorname{ker} T_{f} T_{g} \subset \operatorname{ker} T_{\varphi} \text {. }
$$

Proof. First claim $\operatorname{ker} T_{f} T_{g} \otimes \overline{H^{2}} \neq L^{1}$, where $\operatorname{ker} T_{f} T_{g} \otimes \overline{H^{2}}$ denotes the $L^{1}$-closure of linear manifold \{finite sum $\left.\sum \lambda_{i} h_{i} \overline{h_{i}^{\prime}}: \forall h_{i} \in \operatorname{ker} T_{f} T_{g}, \forall h_{i}^{\prime} \in H^{2}\right\}$. If not, by Lemma 2 there exists a function $\psi(\neq 0)$ such that for any $h \in \operatorname{ker} T_{f} T_{g}$ we have $T_{g} h=\psi h$. Since

$$
\begin{aligned}
\int g\left(\sum \lambda_{i} h_{i} \overline{h_{i}^{\prime}}\right) d \theta & =\sum \lambda_{i}\left\langle T_{g} h_{i}, h_{i}^{\prime}\right\rangle=\sum \lambda_{i}\left\langle\psi h_{i}, h_{i}^{\prime}\right\rangle \\
& =\int \psi\left(\sum \lambda_{i} h_{i} \overline{h_{i}^{\prime}}\right) d \theta
\end{aligned}
$$

it follows that

$$
\left|\int \psi\left(\sum \lambda_{i} h_{i} \overline{h_{i}^{\prime}}\right) d \theta\right| \leq\|g\|_{\infty}\left\|\sum \lambda_{i} h_{i} \overline{h_{i}^{\prime}}\right\|_{L^{1}}
$$

Because the above inequality is true on the dense subset $\operatorname{ker} T_{f} T_{g} \otimes \bar{H}^{2}$ of $L^{1}, M_{\psi}$, determines a bounded linear functional on $L^{1}$. From this, we obtain a $\psi \in L^{\infty}$ and $\psi=g$. By Lemma $1,|\psi|>0$ a.e. This contradicts $\left.g\right|_{E}=0$. It follows that $\operatorname{ker} T_{f} T_{g} \otimes \overline{H^{2}} \neq L^{1}$. We prove the claim.

According to the claim there exists a bounded linear functional which annihilates $\operatorname{ker} T_{f} T_{g} \otimes \overline{H^{2}}$, i.e., there exist $\varphi \in L^{\infty}$ and $\varphi \neq 0$ such that for any $h_{0} \in \operatorname{ker} T_{f} T_{g}$, $h \in H^{2}$, we have

$$
\int \varphi h_{0} \bar{h} d \theta=\left\langle T_{\varphi} h_{0}, h\right\rangle=0 .
$$

Hence $h_{0} \in \operatorname{ker} T_{\varphi}$, and it follows that $\operatorname{ker} T_{f} T_{g} \subset \operatorname{ker} T_{\varphi}$. This completes the proof of Theorem 1. 
To prove Theorem 2, we need the following lemma.

Lemma 3 ([1, p. 179]). If $T_{\varphi_{1}} T_{\varphi_{2}} \cdots T_{\varphi_{n}}=0$, then $\varphi_{1} \varphi_{2} \cdots \varphi_{n}=0$.

Theorem 2. If $T_{\varphi_{1}} T_{\varphi_{2}} T_{\varphi_{3}} T_{\varphi_{4}} T_{\varphi_{5}}=0$, then there exist some $i$ such that $\varphi_{i}=0$.

Proof. Suppose $\operatorname{ker} T_{\varphi_{1}} \neq 0$ and $\operatorname{ker} T_{\overline{\varphi_{5}}} \neq 0$ without loss of generality. Then clearly $\left|\varphi_{1} \varphi_{5}\right|>0$ a.e. By Lemma 3 we have

$$
\varphi_{2} \varphi_{3} \varphi_{4}=0 .
$$

Assume $\varphi_{3} \neq 0$. It follows that there exists a measurable subset $E$ of $T$ with $0<$ meas $E<2 \pi$ such that

$$
\left.\varphi_{2}\right|_{E}=0 \quad \text { or }\left.\quad \varphi_{4}\right|_{E}=0 .
$$

In case $\left.\varphi_{2}\right|_{E}=0$, suppose $T_{\varphi_{3}} T_{\varphi_{4}} T_{\varphi_{5}} \neq 0$; hence $\operatorname{ker} T_{\varphi_{1}} T_{\varphi_{2}} \neq\{0\}$. From Theorem 1 and Rang $T_{\varphi_{3}} T_{\varphi_{4}} T_{\varphi_{5}} \subset \operatorname{ker} T_{\varphi_{1}} T_{\varphi_{2}}$, we know that there exists a $\varphi \in L^{\infty}$, $\varphi \neq 0$, such that

$$
T_{\varphi} T_{\varphi_{3}} T_{\varphi_{4}} T_{\varphi_{5}}=0 .
$$

Since $|\varphi|>0,\left|\varphi_{5}\right|>0$ a.e., by Lemma $3 \varphi_{3} \varphi_{4}=0$. Thus there exists a measurable subset $E^{\prime}$ of $T$ with $0<$ meas $E^{\prime}<2 \pi$ such that $\left.\varphi_{4}\right|_{E^{\prime}}=0$. By $(*)$, we have

$$
T_{\overline{\varphi_{5}}} T_{\overline{\varphi_{4}}} T_{\overline{\varphi_{3}}} T_{\bar{\varphi}}=0 \text {. }
$$

Since $T_{\overline{\varphi_{3}}} T_{\bar{\varphi}} \neq 0$, by Theorem 1 and Rang $T_{\overline{\varphi_{3}}} T_{\bar{\varphi}} \subset \operatorname{ker} T_{\overline{\varphi_{5}}} T_{\overline{\varphi_{4}}}$, there exists a $\varphi^{\prime} \in L^{\infty},\left|\varphi^{\prime}\right|>0$ a.e., such that

$$
T_{\varphi^{\prime}} T_{\overline{\varphi_{3}}} T_{\bar{\varphi}}=0 .
$$

Again by Lemma $3, \varphi_{3}=0$. This contradicts the assumption; thus we obtain

$$
\varphi_{2}=0 \text { or } \varphi_{4}=0 .
$$

Let $\left.\varphi_{4}\right|_{E}=0$, and assume $\varphi_{3} \neq 0$. Similarly, we have

$$
\varphi_{2}=0 \text { or } \varphi_{4}=0 .
$$

Therefore if $T_{\varphi_{1}} T_{\varphi_{2}} T_{\varphi_{3}} T_{\varphi_{4}} T_{\varphi_{5}}=0$, then there exists some $i$ such that $\varphi_{i}=0$. This completes the proof of Theorem 2 .

Using the approach in this note, we may prove the following

Theorem 3. If $T_{\varphi_{1}} T_{\varphi_{2}} T_{\varphi_{3}} T_{\varphi_{4}} T_{\varphi_{5}}$ has finite rank, then there exists some $i$ such that $\varphi_{i}=0$.

\section{ACKNOWLEDGMENTS}

The author would like to thank Professors Sun Shun Hua \& Yu Da Hai for their encouragement and help. He would also like to thank the NNSFC for partial support.

\section{REFERENCES}

1. R. G. Douglas, Banach algebra techniques in operator theory, Academic Press, New York, 1972 MR 50:14335

Department of Mathematics, Sichuan University, Cheng Du, 610064, People's RepubLIC OF CHINA

Current address: Institute of Mathematics, Fudan University, Shanghai 200433, People's Republic of China 